\title{
On the $\beta$ - and quark mass dependence of the nuclear transition in the strong coupling regime
}

\author{
Jangho Kim* \\ Institut für Theoretische Physik, Goethe-Universität Frankfurt am Main, Max-von-Laue-Str. 1, \\ 60438 Frankfurt am Main, Germany \\ E-mail: jkimeth.physik.uni-frankfurt.de
}

\section{Owe Philipsen}

Institut für Theoretische Physik, Goethe-Universität Frankfurt am Main, Max-von-Laue-Str. 1, 60438 Frankfurt am Main, Germany

E-mail: philipseneth.physik.uni-frankfurt.de

\section{Wolfgang Unger}

Fakultät für Physik, Universität Bielefeld, Universitätstasse 25, D33619 Bielefeld, Germany

E-mail: wungerephysik.uni-bielefeld.de

Lattice QCD in a dual formulation with staggered fermions is well established in the strong coupling limit and allows to perform Monte Carlo simulations at finite baryon chemical potential. We have recently addressed the dependence of the nuclear critical end point as a function of the quark mass $a m_{q}$, and separately as a function of the lattice gauge coupling $\beta$ in the chiral limit. Here we proceed to determine the dependence of the nuclear transition on both, $a m_{q}$ and $\beta$, on isotropic lattices and attempt to pinpoint the critical end point for various $\beta$ where the sign problem is still manageable.

37th International Symposium on Lattice Field Theory - Lattice2019

16-22 June 2019

Wuhan, China

${ }^{*}$ Speaker. 


\section{Introduction}

The finite density sign problem hinders the direct Monte Carlo simulation of QCD at finite baryon chemical potential. As an alternative method, we adopt the dual representation which is changing degrees of freedom of the original theory to integer variables. The dual representation in the strong coupling regime allows us to investigate the full $\mu_{B}-T$ phase diagram. We have studied the dependence of the nuclear critical end point (CEP) as a function of the quark mass $a m_{q}$ in the strong coupling limit $(\beta=0)$ [1]. If quark mass increases, the critical baryon chemical potential increases and the critical temperature decreases. Hence, as shown in Fig. 1, the critical end point moves to the bottom right direction. We also have presented the $\beta$ dependence in the chiral limit in our previous study [2]. In these proceedings, we present the $\beta$ dependence of the critical end line at finite quark masses in the strong coupling regime. We sketch the expected behavior of the critical line in Fig. 1. If $\beta$ increases, the critical end point of a certain quark mass is expected to move to lower temperature but the critical baryon chemical potential does not change much. Hence, the first order line shortens with increasing $\beta$.

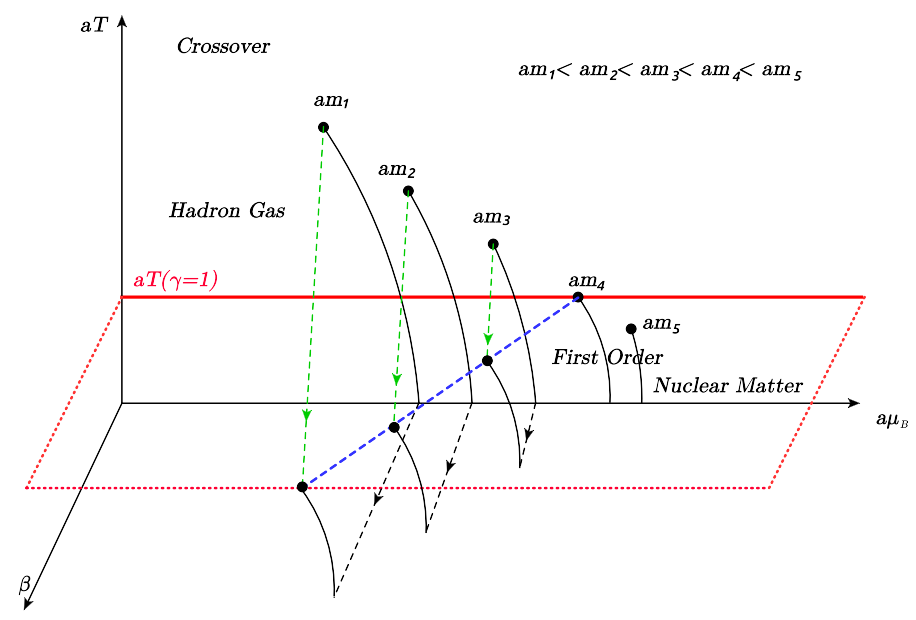

Figure 1: Sketch of $\beta$ and quark mass dependence of the CEP on anisotropic lattices. The red plane denotes fixed temperature in lattice units $a T=1 / N_{t}$, and the dotted blue line is the expected critical end line in the $a T=1 / N_{t}$ plane. The dotted green lines are the expected behavior of CEP at fixed quark mass in $a T>1 / N_{t}$, as a function of $\beta$.

\section{Setup}

We use staggered fermions in the dual formulation $[3,4,5]$ with gauge corrections $O(\beta)$ $[6,2,7]$.

$$
\begin{aligned}
& Z\left(m_{q}, \mu, \gamma\right)= \\
& \sum_{\left\{k, n, \ell, n_{p}\right\}} \underbrace{\prod_{b=(x, \mu)} \frac{\left(N_{c}-k_{b}\right) !}{N_{c} !\left(k_{b}-\left|f_{b}\right|\right) !} \gamma^{2 k_{b} \delta_{\hat{0}, \mu}}}_{\text {singlet hoppings }} \underbrace{\prod_{x} \frac{N_{c} !}{n_{x} !}\left(2 a m_{q}\right)^{n_{x}}}_{\text {chiral condensate }} \underbrace{\prod_{\ell_{3}} w\left(\ell_{3}, \mu\right)}_{\text {triplet hoppings }} \underbrace{\prod_{\ell_{f}} \tilde{w}\left(\ell_{f}, \mu\right)}_{\text {weight modification }} \underbrace{\prod_{P} \frac{\left(\frac{\beta}{2 N_{c}}\right)^{n_{P}+\bar{n}_{P}}}{n_{P} ! \bar{n}_{P} !}}_{\text {gluon propagation }},
\end{aligned}
$$




$$
w\left(\ell_{3}, \mu\right)=\frac{1}{\prod_{x \in \ell_{3}}} \sigma\left(\ell_{3}\right) \gamma^{N_{c} N_{\hat{0}}} \exp \left(N_{c} N_{t} r_{\ell_{3}} a_{t} \mu\right), \quad \sigma\left(\ell_{3}\right)=(-1)^{r_{\ell_{3}}+N_{-}\left(\ell_{3}\right)+1} \prod_{b=(x, \hat{\mu}) \in \ell_{3}} \eta_{\hat{\mu}}(x),
$$

where $k_{b}$ and $f_{b}$ are the number of dimers and gauge fluxes at bond $b, n_{x}$ is the number of monomers at site $x$, and $\ell_{3}$ denotes a 3 -fermion fluxes loop and $\ell_{f}$ is a single fermion loop. $n_{P}$ and $\bar{n}_{P}$ are plaquette (counterclockwise and clockwise) occupation number. $N_{\hat{0}}$ is the number of 3 -fermion fluxes in temporal direction, $r_{\ell_{3}}$ is the winding number in temporal direction. $N_{-}$is the number of 3 -fermion fluxes in negative direction and $\eta_{\hat{\mu}}$ is the staggered phase factor. In this simulation, we fix the temporal lattice extent to $N_{t}=4$ and an anisotropy $\gamma=1$. The lattices temperature is fixed to $a T=\frac{1}{N_{t}}$. If we change $\beta$, the lattice spacing $a$ is changed but $a T$ is invariant. We simulate for $\beta=0.0,0.1, \cdots, 0.9,1.0$ and $a m_{q}$ from 0.0 to 0.5 with step size 0.01 . We scan the baryon chemical potential $a \mu_{B}$ for each $\left(\beta, a m_{q}\right)$ and find the critical baryon chemical potential $a \mu_{c}$. On the $\mu-T$ plane in Fig.1, if the quark mass becomes heavy, the critical end point moves to the large $a \mu$ and low $a T$ region. Because we fix the temperature and vary the quark mass, the first order phase transition occurs at lighter quark masses in our simulation. By contrast, a crossover transition is expected for heavy quark mass. At a certain critical quark mass ( $a m_{4}$ in Fig.1), the transition turns into a second order transition.

\section{Analysis and results}

\subsection{Sign problem}

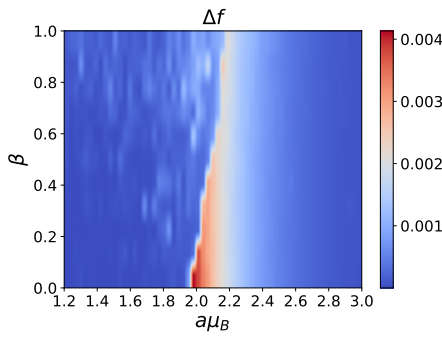

(a) From 3 fermion fluxes

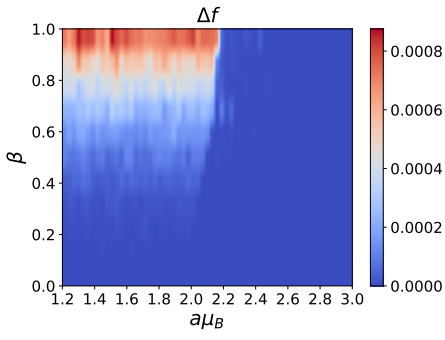

(b) From Single fermion flux

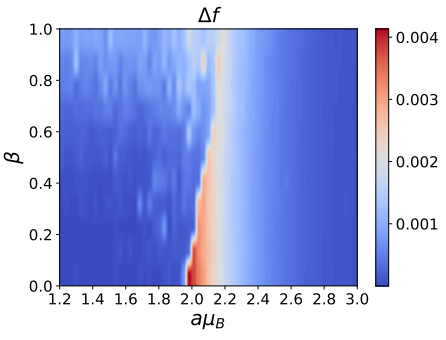

(c) Total sign problem

Figure 2: Sign problems from two difference sources.

First, let us consider the sign problem at finite $\beta$ and $a m_{q}$. The sign problem comes from the odd number of fermion flux. In $O(\beta)$, only single and 3 fermion fluxes cause the sign problem and they are distinguishable. Single fermion flux and 3 fermion fluxes make fermion loops like baryon world lines in the strong coupling limit, and the sign problems is related to their geometries [2]. We distinguish these two types of sign problems and show in the Fig. 2(a) and Fig. 2(b). Here $\Delta f$ is a difference between full and sign quenched free energy density and sign is $\sigma=\exp \left(-N_{s}^{3} N_{t} \Delta f\right)$. In the Fig. 2(a), the sign problem from 3 fermion fluxes mainly occurs near the phase transition region. We will see in the next section that the first order transition weakens when increasing $\beta$. So, the phase transition area gets wide in $a \mu$. The single fermion flux sign problem increases with $\beta$ as expected, but only occurs in the hadronic gas phase as shown in Fig. 2(b). We present the combined sign problem in Fig. 2(c). 


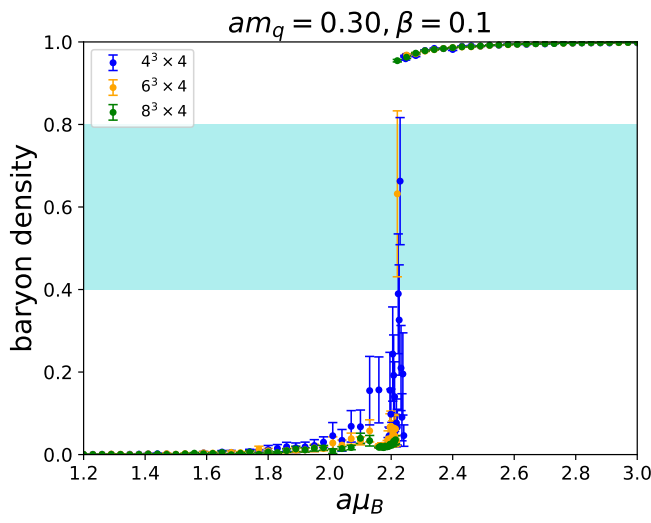

(a) $\beta=0.1, a m_{q}=0.3$

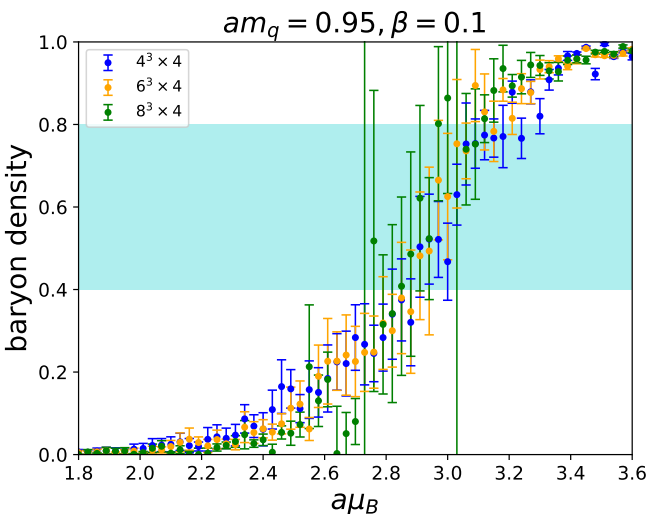

(b) $\beta=0.1, a m_{q}=0.95$

Figure 3: Baryon density at $\beta=0.1$

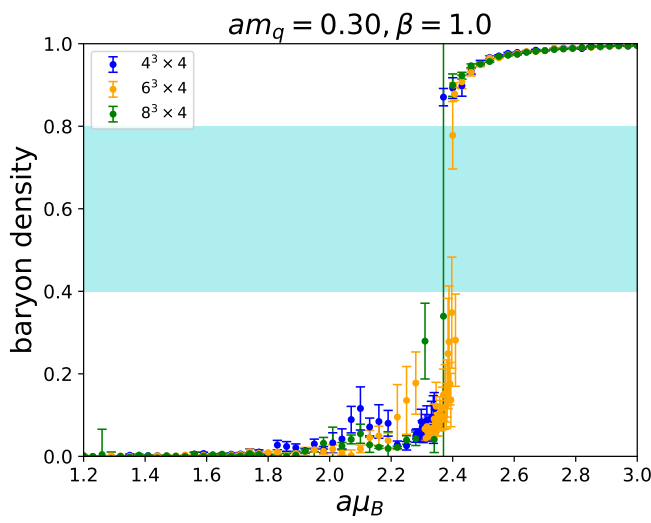

(a) $\beta=1.0, a m_{q}=0.3$

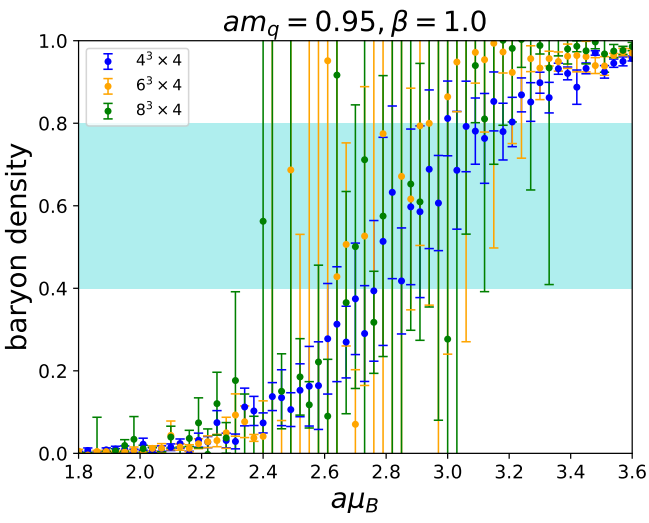

(b) $\beta=1.0, a m_{q}=0.95$

Figure 4: Baryon density at $\beta=1.0$

\subsection{Analysis of baryon density}

Now let us consider the baryon density $\left\langle n_{B}\right\rangle=\frac{1}{V_{s} N_{t}} \frac{\partial}{\partial\left(N_{c} a_{t} \mu\right)} \log Z=\frac{1}{V_{s}}\left\langle\sum_{\ell_{3}} r_{\ell_{3}}\right\rangle$ which is our observable to determine the critical end points, where $V_{s}$ is a spatial volume. We present our results of baryon density in Fig. 3 and Fig. 4. In a first step, the critical $a \mu_{c}$ is determined by data points crossing the blue band, which was chosen by eye to be sufficiently distinct from both zero and saturation. In Fig. 3 we compare the onset of the nuclear transition for $\beta=0.1$ for two different quark masses and various volumes. At small quark mass, the transition is consistent with first order, and large quark mass weakens the phase transition. This also holds for Fig. 4. If we fix the quark mass and change $\beta$, comparing Fig. 3(a) and Fig. 4(a) or Fig. 3(b) and Fig. 4(b), we can see increasing $\beta$ diminishes the phase transition. From the above analysis, we choose the $a \mu_{c}$ and plot them with respect to $a m_{q}$ in Fig. 5. The small quark mass region results in a first order phase transition and the large quark region results in a crossover. As $\beta$ increases, the first order region shrinks and the crossover is extended. Between these first order and crossover transitions, there is 


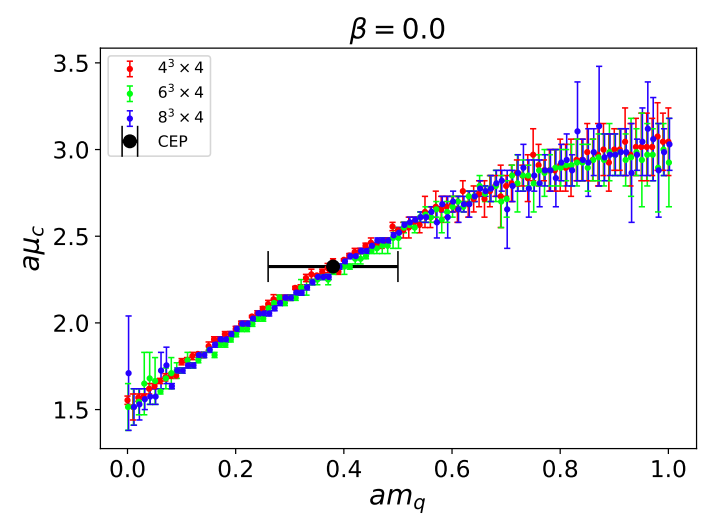

(a) $\beta=0.0$

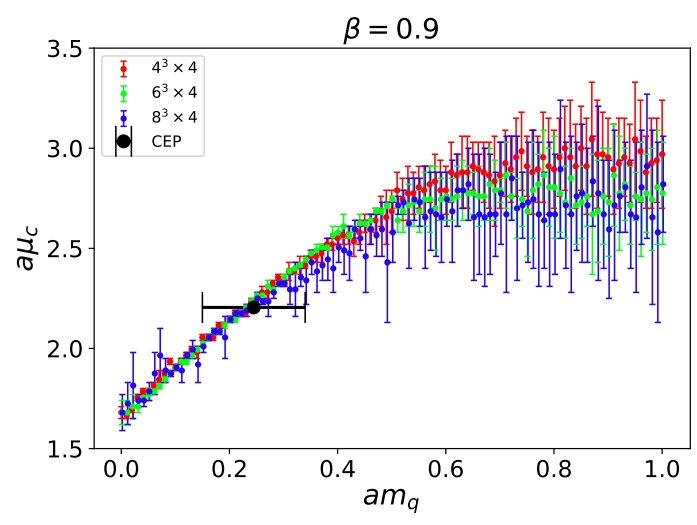

(b) $\beta=0.9$

Figure 5: Critical chemical potential $\left(a \mu_{c}\right)$ as a function of quark mass $\left(a m_{q}\right)$.

a critical end point. Hence, the critical end point is moving to the smaller quark masses when $\beta$ is increased.

\subsection{Critical end point analysis using histograms}
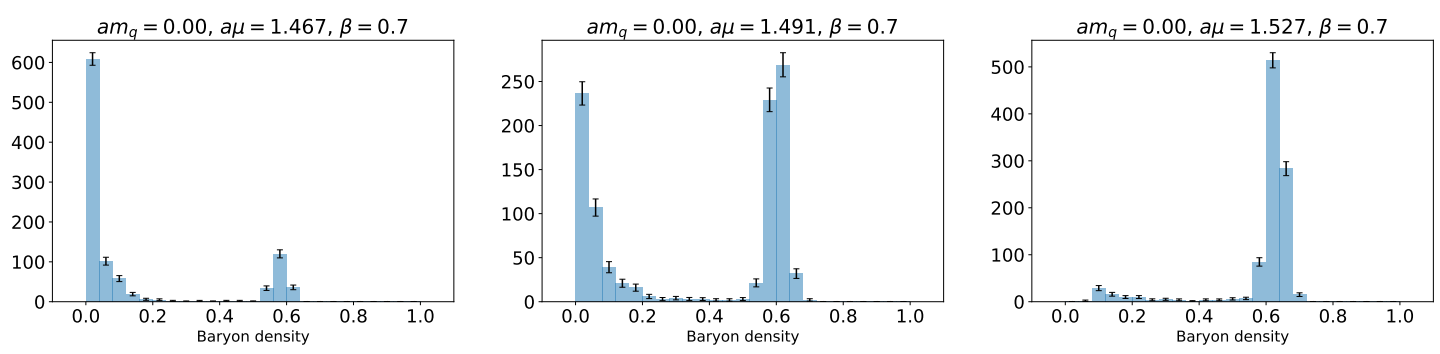

Figure 6: Baryon density histogram at small quark mass $a m_{q}=0.0$
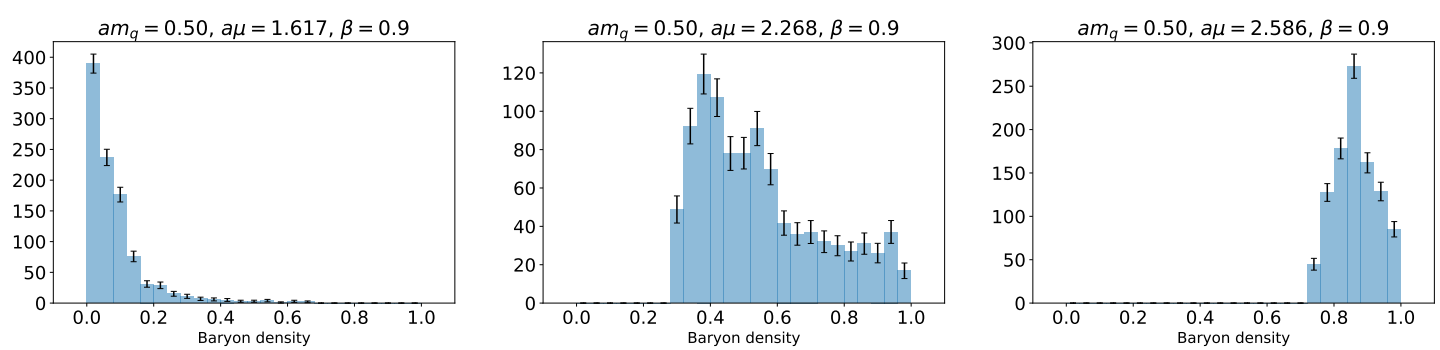

Figure 7: Baryon density histogram at large quark mass $a m_{q}=0.5$

Because of the huge statistical errors near the critical chemical potential $\mu_{c}$, the analysis of the baryon susceptibility is difficult. So, we analyse the data using histograms to bound the critical end points. We use the data of a $8^{3} \times 4$ volume for the histograms and the errors are computed by the bootstrap resampling method. In the Fig. 6 and Fig. 7, we plot histograms around $a \mu_{c}$. At small 


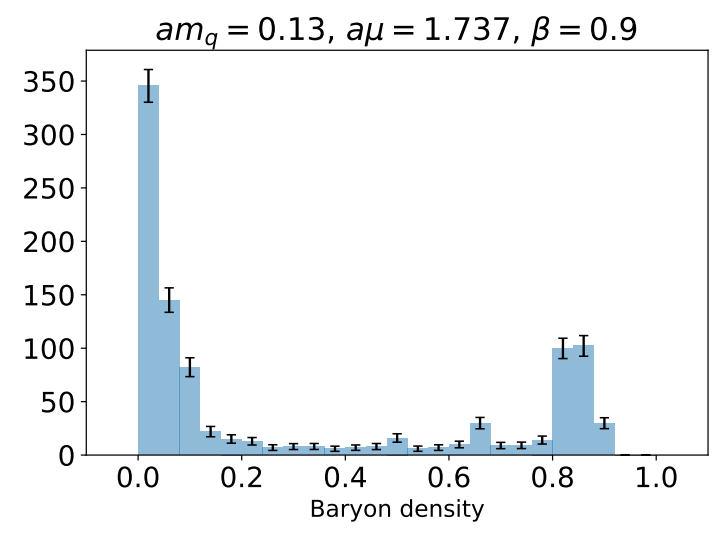

(a) Lower bound on CEP

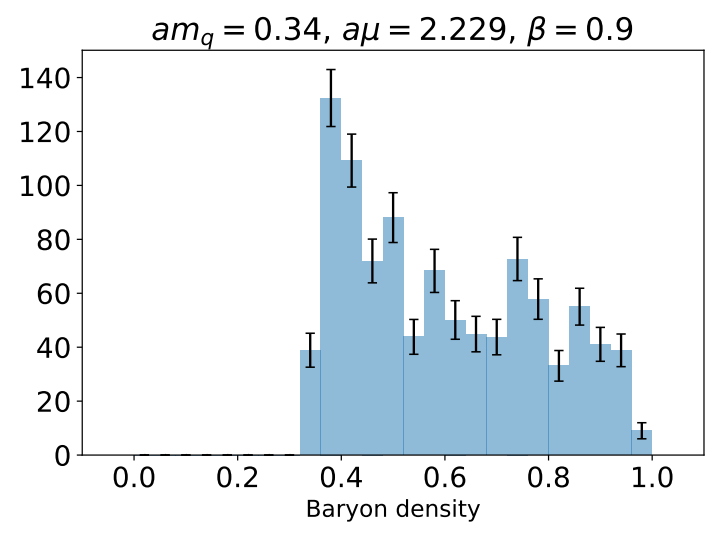

(b) Upper bound on CEP

Figure 8: Baryon density histogram of lower and upper limit of $a m_{q}$ at $a \mu_{c}$ for critical end point at $\beta=0.5$.

quark masses, the histograms have a two-peak distribution. Clearly at this quark mass, the phase transition is of first order. For large quark mass, there is no two-peak distribution and the peak moves smoothly from one state to the other state with increasing $a \mu$. There is no perfect two-peak distributions in the first order transition histogram because of finite volume effects, so we choose the lower bound for the CEP when two-peak distribution is clear that is shown in Fig. 8(a). The upper bound for the CEP is selected when two-peak vanishes and a peak starts to move smoothly. This is presented in Fig. 8(b). From the histogram analysis we can determine the upper limit of quark mass for the first order phase transition and the lower limit of that for crossover. Between those upper and lower limits, the second order end point is located. The critical line as a function of $\beta$ is presented in Fig. 9(a). In this plot, the left lower corner corresponds to the first order region and the right upper corner is crossover. As $\beta$ increases, the quark mass of the CEP decreases slightly. This is similar for the baryon chemical potential as shown in Fig. 9(b). To determine the error bars in Fig. 9(b), we take the smallest and largest quark masses in Fig. 9(a) for each $\beta$. Then there are corresponding $a \mu_{c}$ for quark masses. We use the range of these $a \mu_{c}$ values to the errors of critical line in Fig. 9(b). We determine the error bars from the histograms very conservatively. The huge errors in Fig. 9 are caused by the uncertainty and small statistics. The $\beta$ dependence of both $a m_{q}$ and $a \mu$ are linear in $\beta$ in the small $\beta \lesssim 1$ region.

\section{Conclusion}

We simulate the dual representation with finite quark mass and lattice gauge coupling $\beta$. Because this simulation takes into account only $O(\beta)$, we restrict to the range of $\beta$ smaller than one. In this parameter space, the sign problem is still mild enough to use a sign reweighting method. We obtain the $\beta$ dependence of the critical line using a histogram analysis. In the small $\beta$ region, the critical line looks like linear, but still has large errors. Hence, the higher corrections of $\beta$ are essential to extend this study to $\beta$ larger than one and an important step in this direction has been addressed in Ref. [7]. 


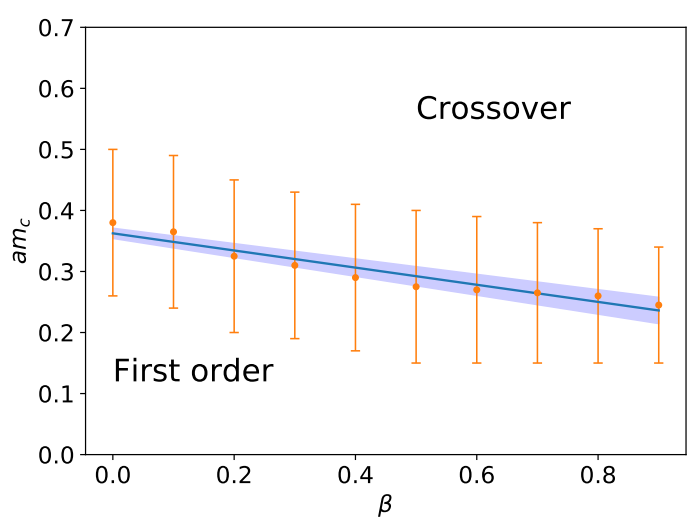

(a) Quark mass critical line

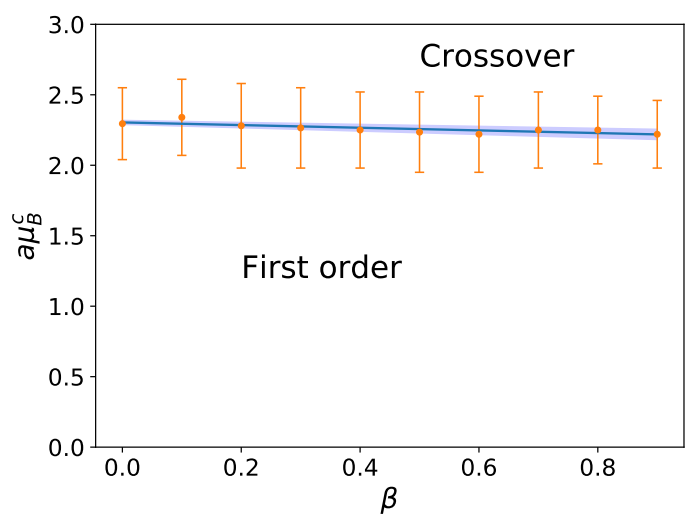

(b) Baryon chemical potential critical line

Figure 9: Critical line of end points as a function of $\beta$.

\section{Acknowledgments}

The authors J.K., O.P. and W.U. acknowledge support by the Deutsche Forschungsgemeinschaft (DFG, German Research Foundation) through the CRC-TR 211 'Strong-interaction matter under extreme conditions'- project number 315477589 - TRR 211. J.K. would like to thank the Center for Scientific Computing, University of Frankfurt for making their High Performance Computing facilities available. W.U. acknowledges support by the Deutsche Forschungsgemeinschaft (DFG) through the Emmy Noether Program under Grant No. UN 370/1.

\section{References}

[1] J. Kim and W. Unger, Quark Mass Dependence of the QCD Critical End Point in the Strong Coupling Limit, PoS LATTICE2016 (2016) 035, [1611.09120].

[2] G. Gagliardi, J. Kim, and W. Unger, Dual Formulation and Phase Diagram of Lattice QCD in the Strong Coupling Regime, EPJ Web Conf. 175 (2018) 07047, [1710 . 07564].

[3] P. Rossi and U. Wolff, Lattice QCD With Fermions at Strong Coupling: A Dimer System, Nucl. Phys. B248 (1984) 105-122.

[4] D. H. Adams and S. Chandrasekharan, Chiral limit of strongly coupled lattice gauge theories, Nucl. Phys. B662 (2003) 220-246, [hep-lat/ 0303003$].$

[5] M. Fromm, Lattice QCD at string coupling: thermodynamics and nuclear physics, Thesis (2010).

[6] P. de Forcrand, J. Langelage, O. Philipsen, and W. Unger, Lattice QCD Phase Diagram In and Away from the Strong Coupling Limit, Phys. Rev. Lett. 113 (2014), no. 15 152002, [1 406 . 4397].

[7] G. Gagliardi and W. Unger, A new dual representation for staggered lattice QCD, 1911.08389. 